\title{
Effect of ECG derived respiration (EDR) on modeling ventricular repolarization dynamics in different physiological and psychological conditions
}

\author{
MH Imam ${ }^{1}$, CK Karmakar ${ }^{1 *}$, AH Khandoker ${ }^{1,2}$, M Palaniswami $^{1}$ \\ ${ }^{1}$ Department of Electrical and Electronic Engineering, The University of \\ Melbourne, Parkville, Melbourne, VIC 3010, Australia. \\ 2 Department of Biomedical Engineering, Khalifa University of Science, \\ Technology and Research, Abu Dhabi, UAE.
}

+61-(0)3-90357614

$+61-(0) 3-83446678$

karmakar@unimelb.edu.au

Total number of words: 6865

Number of words in abstract: 331

Number of Figures: 5

Number of Tables: 1

\section{Abstract}

Ventricular repolarization dynamics is an important predictor of the outcome in cardiovascular diseases. Mathematical modeling of the heart rate variability ( $R R$ interval variability) and ventricular repolarization variability ( $Q T$ interval variability) is one of the popular methods to understand the dynamics of ventricular repolarization. Although ECG derived respiration (EDR) was previously suggested as a surrogate of respiration but effect of respiratory movement on ventricular repolarization dynamics was not studied. In this study, the importance of considering the effect of respiration and the validity of using EDR as a surrogate of respiration for linear parametric modeling of ventricular repolarization variability is studied in two cases with different physiological and psychological conditions. In the first case study, we used 20 young and 20 old healthy subjects' ECG and respiration data from Fantasia database at Physionet to analyse a bivariate $Q T-R R$ and a trivariate $Q T-R R-R E S P$ or $E D R$ model structure to study the ageing effect on cardiac repolarization variability. In the second study, we used 16 healthy subjects' data from drivedb (stress detection for automobile drivers) database at Physionet to do the same analysis for different psychological condition (i.e. in stressed and no stress condition). The results of our study showed that model having respiratory information $(Q T-R R-R E S P$ and $Q T-R R-E D R)$ gave significantly better fit value $(\mathrm{p}<0.05)$ than that of found from the $Q T-$ 
$R R$ ) model. EDR showed statistically similar ( $>0.05)$ performance as that of respiration as an exogenous model input in describing repolarization variability irrespective of age and different mental conditions. Another finding of our study is that both respiration and EDR based models can significantly $(\mathrm{p}<0.05)$ differentiate the ventricular repolarization dynamics between healthy subjects of different age groups and with different psychological conditions whereas models without respiration or EDR cannot distinguish between the groups. These results established the importance of using respiration and the validity of using EDR as a surrogate of respiration in the absence of respiration signal recording in linear parametric modeling of ventricular repolarization variability in healthy subjects.

\section{Keywords}

ECG derived respiration, heart rate variability, ventricular repolarization variability, repolarization dynamics, linear parametric model, respiration, stress, ageing

\section{Introduction}

Modeling heart rate (HR) variability (i.e. $R R$ interval variability) and ventricular repolarization (VR) variability (i.e. $Q T$ interval variability) is a non-invasive estimation of the dynamic properties of the cardiovascular system. Although, both HR and VR are controlled predominantly by autonomic nervous system (ANS) [2], VR variability (VRV) is also directly affected by the HR variability (HRV) and other factors like respiration [24,26]. Respiration directly affects HRV through respiratory sinus arrhythmia (RSA) [26,4] and it also affect VRV due to direct relation between VRV and HRV. Hanson et al.[9] has also reported the presence of cyclic modulation of VR duration due to respiration in healthy human. Therefore, respiration signal should be added in modeling $Q T$ and $R R$ interactions for better comprehension of ventricular repolarization dynamics.

Traditional respiration signal recording procedures (e.g. impedance pneumography, spyrometery etc.) were not very much suited in cases like ambulatory monitoring and sleep study due to the necessity of using bulky and expensive recording devices which might affect the natural breathing process of the subject $[3,5]$. Since the cost and complexity associated with respiratory signal acquisition restricts the recording of respiratory information in every situation, a lot of research has been conducted to devise and validate a surrogate for respiratory movement from other available physiological signals. Among others, 
ECG signal has been highly used to extract respiratory movement in ambulatory settings. The ECG derived respiration (EDR) has been successfully used as a surrogate for respiration in various studies with different physiological and pathological conditions $[3,7,15,20]$ and in daily dynamic activity monitoring from ambulatory single lead ECG [5]. However, to our knowledge EDR has never been used as a surrogate of respiration in modeling VR dynamics.

Porta et al. $[24,26]$ first proposed a linear dynamic autoregressive model from short length ECG data describing VR dynamics using $R R$ and $Q T$ interval (both $\mathrm{QT}_{\text {peak }}$ and $\mathrm{QT}_{\text {end }}$ intervals) interactions. They used $R T$ interval ( $R$ wave to $T$ wave peak or end intervals) instead of $Q T$ interval due to problems in proper detection of $Q$ wave and $T$ wave end in describing the VR duration variability [24,26]. Almeida et al. [2] have used the same model structure reported by Porta et al. [24] except for using $\mathrm{QT}_{\text {end }}$ interval instead of $R T$ interval to describe the effect of heart rate and autonomic nervous system on VR dynamics. These studies validated the use of linear autoregressive models to describe the VR dynamics for short length ECG segments (i.e. 5 min ECG) where the $R R$ and $Q T$ intervals are assumed to be stationary within the data length.

The main objectives of our study are: (i) to investigate the effect of respiration on modeling VRV and the validity of using EDR as an exogenous input in place of original respiration signal in $Q T-R R$ modeling; and (ii) to check whether EDR shows the similar effect as that of respiration on VR dynamics with ageing and with stress induction in healthy subjects. We hypothesize that respiration signal should be added for better comprehension of VR dynamics and EDR can be used as a surrogate of respiration for modeling VR dynamics where respiration signal is not available. To validate our hypotheses we designed two case studies using two databases available at Physionet (i.e. Fantasia and drivedb). We used both $\mathrm{QT}_{\text {peak }}$ and $\mathrm{QT}_{\text {end }}$ intervals in both studies to design the models for understanding the respiratory effect in improving the model performance to describe VR dynamics. 


\section{Data and Methods}

\subsection{Subjects}

To investigate the effect of respiration and to check whether EDR can describe cardiac repolarization dynamics with the variation of age in the first case study, a total of 20 young (21-34 years) and 20 old subjects' (68-85 years) ECG and respiration signals were collected from Fantasia database available at Physionet [8]. In both Young and old groups, equal numbers of male and female (i.e. 10 male and 10 female) subjects were recruited for data collection with no history of cardiovascular diseases. From the 120 mins of simultaneous recording of Lead II ECG and respiration, we used 5 minutes of ECG and respiration signals for extracting the $R R, Q T$ and EDR time series for modeling. 5 minutes section was selected such that $R$ and $T$ waves of ECG were clearly visible and detectable within the selected segment and the respiration signal was free from any visible movement artifacts. Subjects were in supine resting state in sinus rhythm and watching the movie Fantasia (Disney, 1940) to remain awake during the data recording period. Respiratory movement was recorded using the thoracic belt. Both ECG and respiration signals were sampled at $250 \mathrm{~Hz}$.

For the second study, the ECG and respiration signals were taken from Stress Recognition in Automobile Drivers (drivedb) database available at Physionet [8] to understand the $Q T-R R$ interactions in changed psychological conditions. From this database, a total of 16 healthy subjects' data were taken out of 17 subjects' recordings. One recording (drive01) was dropped from our study due to problem in proper detection of $T$ wave parameters. Using this database, an experimental protocol was designed and verified for the detection of stress using various physiological signals (e.g. Electrocardiogram (ECG), Electromyogram (EMG), skin conductivity and respiration), which was induced due to driving in heavy traffic condition. The total drive period consisted of rest, highway and city driving with heavy traffic which were assumed to induce low, medium and high level of stress in the driver's mind. The details of this study protocol i.e. driving protocol, driving period, stress measurement and validation of stress level assessment techniques etc. were described in details by Healy et al. [10]. In this study, we have used 5 minutes ECG and respiration signals segment during 
resting (i.e. No Stress) and city driving (i.e. Highly Stressed) conditions. Recordings of resting condition were treated as data for stable physiological condition and grouped as "No stress", whereas recordings of city driving condition were considered as stressed condition data and grouped as "Stressed". ECG signals were recorded with a modified lead II configuration and sampled at 496Hz. The respiration signals were recorded with an elastic Hall Effect sensor by measuring the chest cavity expansion of the subject at $31 \mathrm{~Hz}$ sampling frequency. The detail procedure of signal collection and analysis was described in [10].

\subsection{ECG and respiratory signal parameter extraction}

ECG signal was first filtered with a median filter to remove baseline wandering. The $R R$ and $Q T$ interval time series were formed by detecting the $R$ wave peak, $Q$ wave onset, $T$ wave peak and $T$ wave end from the ECG signal. Different wave components and intervals of ECG signal used in this study are shown in Figure1.

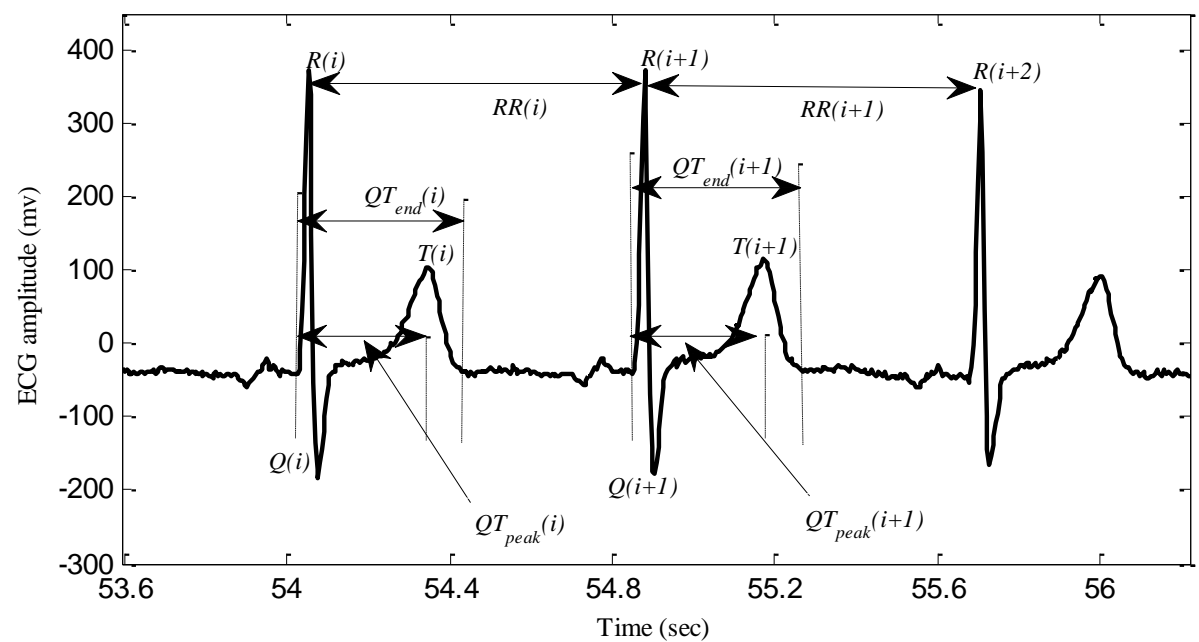

Figure 1: Different ECG wave components $(Q, R$, and $T$ waves) and measurement of $R R$ and $Q T$ intervals $\left(\mathrm{QT}_{\text {peak }}\right.$ and $\left.\mathrm{QT}_{\mathrm{end}}\right)$ for three cardiac beats.

The $R R$ interval was found from the difference between two consecutive $R$ wave peaks which are detected by an algorithm for detecting $Q R S$ complex proposed by Pan et al. [22]. The $Q$ wave onset (i.e. $Q$ point) is determined by detecting the time instant where the gradient of the $Q R S$ complex becomes negative to the left of the $R$ wave peak. The peak of the $T$ wave was detected by searching for the highest point after the $R$ wave. The $T$ wave end or offset is found by searching for the point where the gradient of the $T$ wave first changes its sign after the 
occurrence of $T$ wave peak. This method of detecting the end of the $T$ wave is similar to the maximum slope intercept method, which defines the end of the $T$ wave as the intercept between the isoelectric line with the tangent drawn through the maximum down slope of the $T$ wave $[30,31]$. We used both $\mathrm{QT}_{\text {peak }}$ (i.e. interval from the $Q$ wave onset to the peak of $T$ wave) and $\mathrm{QT}_{\text {end }}$ (i.e. interval from $Q$ wave onset to the end of $T$ wave) intervals to build and validate the model performance. Ectopic beats were removed from $R R$ interval series before used in modeling using the criteria used by Huikuri et al. [11] though the number of ectopic beats is negligible in the recordings. Variations in $R R$ interval time series were also checked by the criteria proposed by Clifford et al. [6] to remove rapid fluctuations, which affect the linear property of the $R R$ series required for the model formation. QT intervals outside the range of 3-SD band were rejected for the formation $Q T$ interval time series, which is the model output signal. This was done for maintaining the stationarity in the input and output time series data for this linear model analysis. The respiration signal time series (RESP) for the model was formed by sampling the continuous respiratory signal recording at each $R$ peak of the ECG. For the derivation of EDR, we used the $R$ wave amplitude method for single lead ECG. In this method, first the ECG signal is filtered for baseline wandering by a median filter. Then from the baseline corrected ECG, $Q R S$ wave were detected using Pan Tompkin's algorithm [22] and the amplitude of the $R$ wave peaks from the detected $Q R S$ wave were recorded to generate the EDR wave time series [14,20]. The sampled respiration (RESP) and EDR calculation technique is graphically illustrated in figure 2 . 

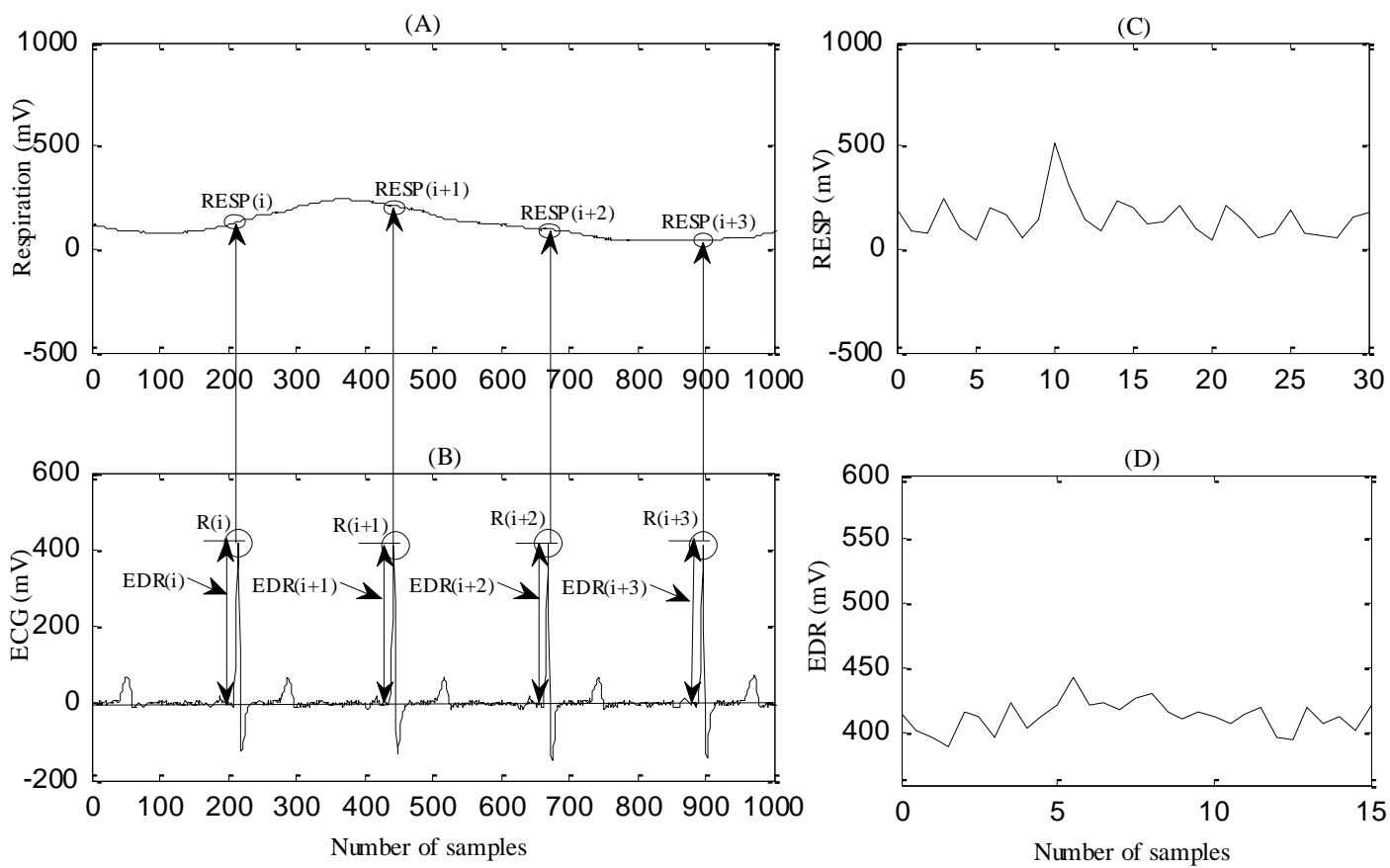

Figure 2: Derivation of RESP (sampled respiration signal) and EDR (ECG derived respiration) signal time series from the respiration and baseline corrected ECG signal. (A) shows that RESP(i) signal which is formed from the sampled values of respiration signal collected by thoracic belt at every $\mathrm{R}(\mathrm{i})$ that is the magnitude of $R$ wave at every $R$ wave peak location and $\mathrm{i}=1,2 \ldots . \mathrm{n}$ where $\mathrm{n}$ is the number of ECG $R$ peaks. (B) EDR(i) waveform is calculated from the variation of $R$ wave peak amplitudes, R(i) in the baseline filtered ECG waveform. (C) and (D) shows a sample segment of both the RESP and EDR time series used as model input parameters.

\subsection{Linear parametric model formation}

Due to subjective variation of $Q T-R R$ relation [19], each subject in this study was modeled individually using the fixed model structure (i.e. ARXAR or ARXXAR) by varying the model order to measure the model performance in predicting $Q T$ interval. First 250 consecutive beats of the derived $R R, Q T$ (both $\mathrm{QT}_{\text {peak }}$ and $\mathrm{QT}_{\text {end }}$ ), RESP and EDR time series from five minute ECG segment were used for the formation of the autoregressive models. $Q T, R R$, sampled respiration (RESP) and EDR time series data were linearly detrended by subtracting mean and dividing by the standard deviation before using as model input and output parameters. We first analysed $\mathrm{ARX}_{\mathrm{RR}} \mathrm{AR}$ model, a bivariate single input (i.e. $R R$ is the single exogenous input) single output (SISO) model with an autoregressive noise without any respiration signal to study $Q T$ dynamics. Then two more trivariate multi input single output (MISO) type models $\left(\mathrm{ARX}_{\mathrm{RR}} \mathrm{X}_{\mathrm{RESP}} \mathrm{AR}\right.$ and $\left.\mathrm{ARX}_{\mathrm{RR}} \mathrm{X}_{\mathrm{EDR}} \mathrm{AR}\right)$ were derived using the methodology 
developed by Porta et al. [26] with $R R$ and RESP or EDR were used as two exogenous inputs. Figure 3 shows the basic ARXXAR type model structure used in the analysis. $R R$ and respiration signals (RESP and EDR) were used as the exogenous inputs in these models. The beat to beat intervals are represented as $Q T=\{\mathrm{QT}(\mathrm{i}), \mathrm{i}=1,2, \ldots \ldots \mathrm{N}\}, R R=\{\mathrm{RR}(\mathrm{i}), \mathrm{i}=1,2, \ldots \ldots \mathrm{N}\}, \operatorname{RESP}=$ $\{\operatorname{RESP}(\mathrm{i}), \mathrm{i}=1,2, \ldots \ldots \mathrm{N}\}$, and $\operatorname{EDR}=\{\operatorname{EDR}(\mathrm{i}), \mathrm{i}=1,2, \ldots \ldots \mathrm{N}\}$ where $\mathrm{N}$ is total number of beats used for building the model, in this study $\mathrm{N}=250$. The $\mathrm{i}^{\text {th }} \mathrm{QT}_{\text {peak }}$ or $\mathrm{QT}_{\text {end }}$ interval followed the $\mathrm{i}^{\text {th }} R R$ interval, thus directly linking the present $Q T$ interval with the preceding $R R$ interval. The $\mathrm{i}^{\text {th }}$ respiratory sample $\mathrm{RESP}(\mathrm{i})$ was taken as the sampled value of the respiration signal at every $R$-wave peak.

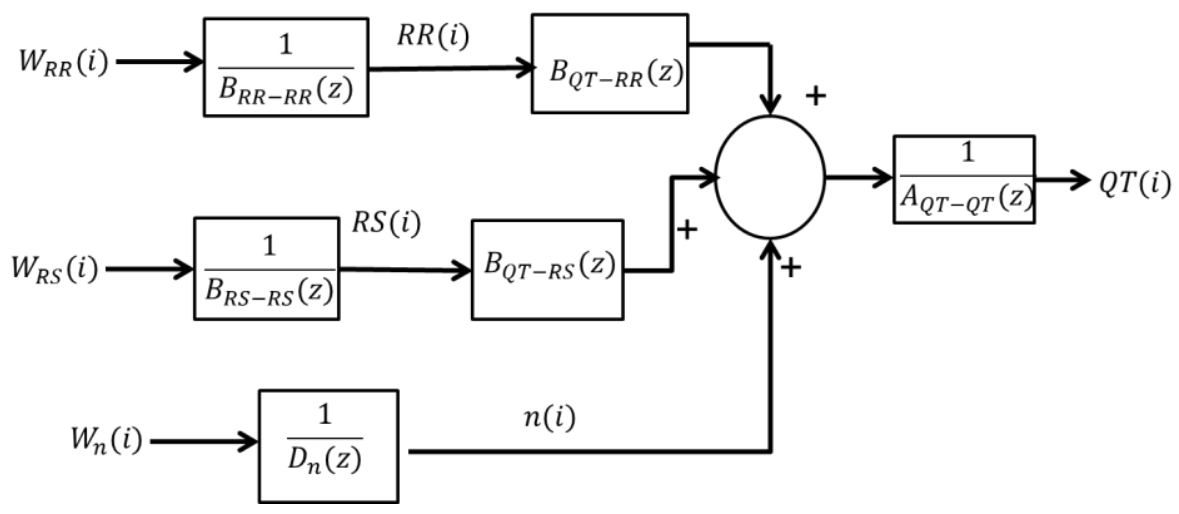

Figure 3: Linear autoregressive parametric model structure with multiple exogenous inputs. RS(i) may be RESP(i) or EDR(i) according to the type of the model.

The equation of the predicted $Q T$ interval for the bivariate $Q T-R R$ model is:

$Q T(i)=A_{Q T-Q T}(z) * Q T(i)+B_{Q T-R R}(z) * R R(i)+n(i)$

and the predicted $Q T$ of the trivariate $Q T-R R-R S$ model including RESP and EDR are defined as:

$$
\begin{aligned}
& Q T(i)=A_{Q T-Q T}(z) * Q T(i)+B_{Q T-R R}(z) * R R(i)+B_{Q T-R e s p}(z) * R E S P(i)+n(i) \\
& Q T(i)=A_{Q T-Q T}(z) * Q T(i)+B_{Q T-R R}(z) * R R(i)+B_{Q T-E D R}(z) * E D R(i)+n(i)
\end{aligned}
$$

Where $R S(i)$ will be RESP(i) or EDR(i) according to model input type. The model performance was validated using both $\mathrm{QT}_{\text {peak }}$ and $\mathrm{QT}_{\text {end }}$ in place of QT(i). The model equations (i.e. equation (1), (2) and (3)) indicate that $i^{\text {th }} Q T$ interval depends on previous $Q T$ intervals, current and previous $R R$ intervals, current and 
past RESP samples and other unknown inherent factors independent of $R R$ and RESP, which were modelled by the noise term $n(i) . A$ and $B$ represent the model transfer function polynomials which actually indicate the memory effect of $Q T$, $R R$, and RESP signals that shows how a $Q T$ interval is affected by current and previous $Q T$ intervals, $R R$ intervals, respiration and other unknown factors (i.e. direct modulatory effect of autonomic nervous system and any other factor that affect $Q T$ other than $R R$ or RESP). The model polynomial coefficients are defined using the following equations:

$$
\begin{aligned}
& A_{Q T-Q T}(z)=\sum_{k=1}^{p} a_{Q T-Q T}(k) * z^{-k} \\
& B_{Q T-R R}(z)=\sum_{k=0}^{p} b_{Q T-R R}(k) * z^{-k} \\
& B_{Q T-R E S P}(z)=\sum_{k=0}^{p} b_{Q T-R E S P}(k) * z^{-k} \\
& B_{Q T-E D R}(z)=\sum_{k=0}^{p} b_{Q T-E D R}(k) * z^{-k}
\end{aligned}
$$

Here $a_{Q T-Q T}(k), b_{Q T-R R}(k)$ and $b_{Q T-R E S P \text { or EDR }}(k)$ are $p, p+1$ and $p+1$ constant coefficients and they were calculated using the system identification techniques[17]. $z^{-k}$ is the $k$ lag delay operator in $z$ domain and $p$ is the identified model order which represents the model complexity for simulation. The larger the value of $p$, the more complex is the model structure to identify the interaction of the system parameters.

The autoregressive noise term is identified by the following equation:

$$
n(i)=D_{n}(z) * n(i)+w_{n}(i)
$$

$$
\text { where, } \quad D_{n}(z)=\sum_{k=1}^{p} d_{n}(k) * z^{-k}
$$

and $w_{n}$ is the zero mean white noise.

The one step ahead prediction error $\left(e r r_{Q T}\right)$ of the model is calculated from the difference between the $Q T(i)$ and best one step ahead predicted $Q T(i)$ of the derived model denoted as $\widehat{Q T}(i)$. The equation of $\operatorname{err}_{Q T}$ is defined by

$e r r_{Q T}=Q T(i)-\widehat{Q T}(i)$

The value of prediction error for the trivariate model incorporating respiration will be as below:

$$
\begin{aligned}
& \operatorname{err}_{Q T}=Q T(i)-\widehat{Q T}(i) \\
& =\left[1-\widehat{D_{n}}(z)\right] *\left[1-\widehat{A_{Q T-Q T}}(z)\right] * Q T(i)-\left[1-\widehat{D_{n}}(z)\right] * \widehat{B_{Q T-R R}}(z) * \\
& \quad R R(i)-\left[1-\widehat{D_{n}}(z)\right] * \widehat{B_{Q T-R S}}(z) * R S(i)
\end{aligned}
$$


where, $R S(i)$ will be RESP(i) or EDR(i) according to the model input type and $\widehat{D_{n}}(z), \widehat{A_{Q T-Q T}}(z), \widehat{B_{Q T-R R}}(z)$ and $\widehat{B_{Q T-R S}}(z)$ are the model transfer function polynomials estimated from the input output data via system identification procedure.

The model goodness of fit value is calculated from the mean squared prediction error (MSPE) which measures the ability of the model structure in fitting the data using the following equations:

$\operatorname{MSPE}=\frac{1}{N} \sum_{i-1}^{N} \operatorname{err}_{Q T}^{2}(i)$

The value of MSPE varies between 0(i.e. perfect fit) and 1(i.e. model cannot fit the data at all).The goodness of fit is defined as

Goodness of fit $=1-\mathrm{MSPE}$

and the higher value of the goodness of fit indicates the higher model prediction capability.

\subsection{Model parameter identification and validation}

The coefficients of the model transfer function polynomials (i.e. $A$ and $B$ ) were calculated using Prediction Error Estimation Method (PEM) for linear models [17]. This method uses a numerical optimization technique to minimize the weighted norm of prediction error, which is defined as the cost function. Model prediction capability is determined by the mean squared prediction error, which is calculated from the difference between measured output and the one step ahead predicted output of the model. Same model order of $p$ was considered in input and output polynomials for calculating the coefficients of the model transfer function which simplifies the model analysis. The range of model order variation was fixed for all subjects and within that range, best model order was selected in terms of AIC criteria [1]. Model order was varied from 9 to 19 to find the best model with lowest value of AIC. Residual analysis was performed on the selected best model according to AIC to check if the model passed the whiteness test and independence test to clarify that model residuals were uncorrelated with each other and not correlated with past input values[17]. In our study $99 \%$ confidence interval was used for the residual test with lag 25 and the model passes the test if the residuals were found uncorrelated (i.e. residual autocorrelation function is within the confidence interval of the estimated response) and residuals were 
found to be uncorrelated with past inputs (i.e. cross correlation between residuals and previous inputs was inside the confidence interval with less than 3 points outside the confidence interval). All these analysis were done using system identification toolbox in MATLAB R2012b.

\subsection{Statistical analysis}

Wilcoxon rank sum test were performed to check the difference between model fitting values of the two groups in two databases. Non-parametric version of one way ANOVA (i.e. Kruskalwallis test) was used to check the statistical difference between three types of model fit values in each database. Then Bonferroni post hoc tests were performed to compare pairwise differences for the three model types. A value of $p<0.05$ was considered significant. All the statistical calculations were carried out in MATLAB R2012b.

\section{Results}

In this study, the goodness of fit values of the SISO and MISO autoregressive models were calculated for two populations of different ages with no cardiovascular abnormalities (i.e. Young and Old groups) in Fantasia database. Same analysis was done for a young subject group with two different psychological conditions available in drivedb database. The variations of model fit for $\mathrm{QT}_{\text {peak }}$ dynamics models in two databases are shown graphically in Figure 4. In both groups of the two databases, it was found that the model predictability (i.e. goodness of fit) increases significantly with the addition of respiration information (RESP or EDR) as an exogenous input in comparison to that found from the model developed using only $Q T$ and $R R$ signals. Similar results were found in $\mathrm{QT}_{\text {end }}$ dynamics models and shown in Figure 5. The trivariate models $\left(\mathrm{ARX}_{\mathrm{RR}} \mathrm{X}_{\mathrm{RESP}} \mathrm{AR}\right.$ and $\left.\mathrm{ARX}_{\mathrm{RR}} \mathrm{X}_{\mathrm{EDR}} \mathrm{AR}\right)$, which had RESP and EDR signals as respiration signal input along with $Q T$ and $R R$, showed statistically similar goodness of fit values for both $\mathrm{QT}_{\text {peak }}$ and $\mathrm{QT}_{\text {end }}$ dynamics models and both are significantly improved with respect to the bivariate $\left(A R X_{R R} A R\right)$ model. These results proved that respiration has a significant effect on VR, which should be considered for modeling VR dynamics. 


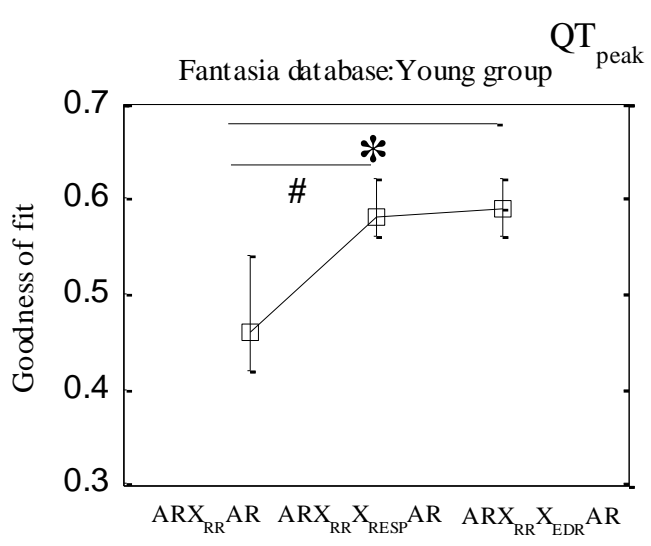

dynamics Model
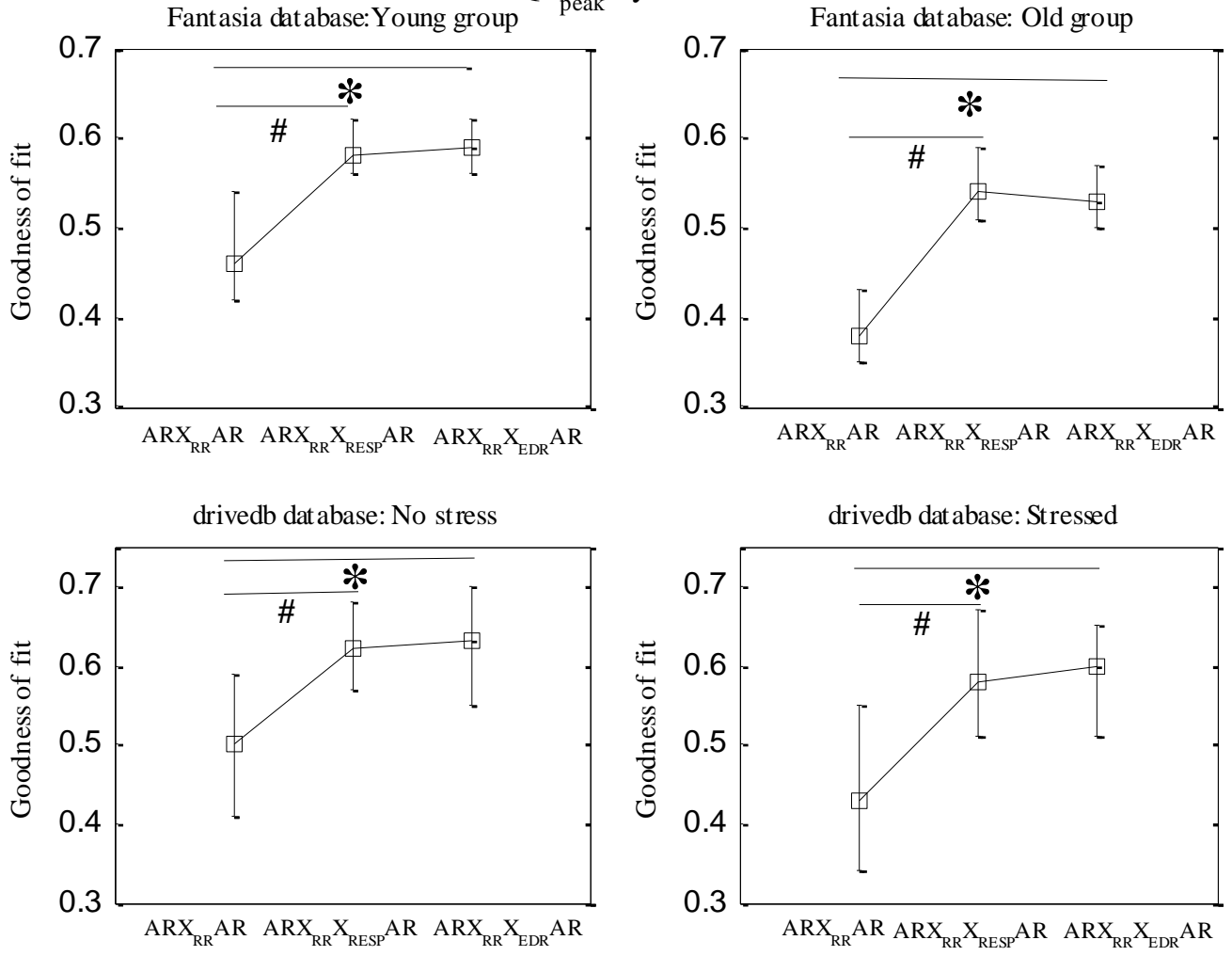

Figure 4: Goodness of fit variation of the analysed $\mathrm{QT}_{\text {peak }}$ dynamics models in Fantasia and drivedb Database at Physionet. \#indicates the significant differences of model fit between $\mathrm{ARX}_{\mathrm{RR}} \mathrm{AR}$ and $\mathrm{ARX} \mathrm{RR}_{\mathrm{RESP}} \mathrm{AR}$ model and * indicates the significant model fit differences between $\mathrm{ARX}_{\mathrm{RR}} \mathrm{AR}$ and $\mathrm{ARX} \mathrm{XR}_{\mathrm{RR}} \mathrm{X}_{\mathrm{EDR}} \mathrm{AR}$ models.

Table 1 demonstrated the effect of using EDR instead of respiration in both databases. These results show that there is no statistically significant difference in model predictability whether it has respiration or EDR as a model input. This validates the use of EDR in modeling $Q T-R R$ interactions in the absence of respiration signal and the effects of respiration and EDR were found almost same on VRV in this modeling study. Another interesting finding of this type of parametric models is that it can also differentiate the effect of ageing and alteration of mental state due to stress induction on the cardiovascular system dynamics. Models describing $\mathrm{QT}_{\text {peak }}$ variability can differentiate the young and elderly group in Fantasia database and in drivedb database it was found that $\mathrm{QT}_{\mathrm{end}}$ dynamics models can significantly differentiate the changes in ventricular repolarization variability with the induction of stress. 


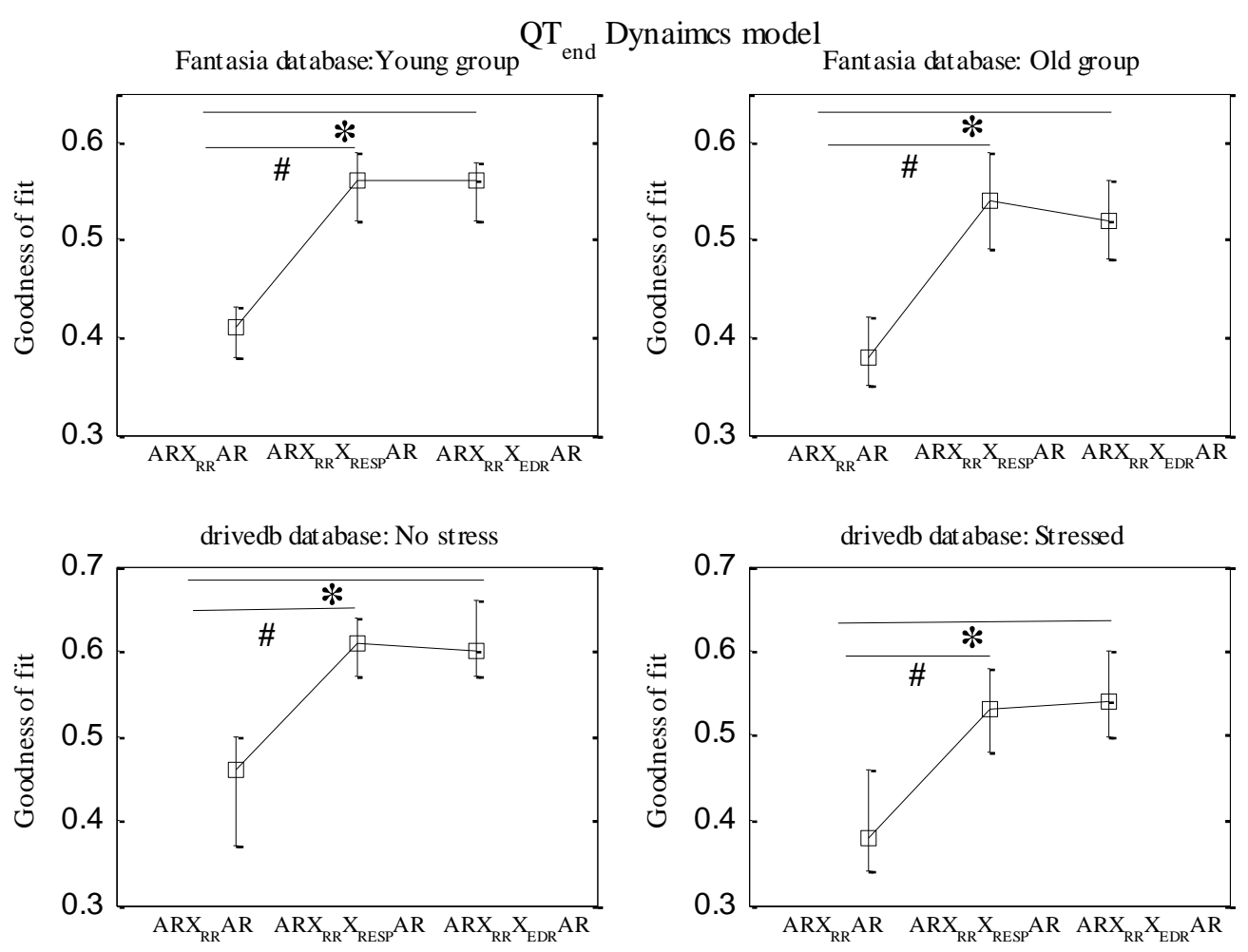

Figure 5: Goodness of fit variation of the analysed $\mathrm{QT}_{\text {end }}$ dynamics models in Fantasia and drivedb Database at Physionet. \# and * indicate the significant differences of model fit between $\mathrm{ARX}_{\mathrm{RR}} \mathrm{AR}$ and $\mathrm{ARX} \mathrm{XR}_{\mathrm{RR}} \mathrm{X}_{\mathrm{RESP}} \mathrm{AR}$ model and between $\mathrm{ARX} \mathrm{XR}_{\mathrm{RR}} \mathrm{AR}$ and $\mathrm{ARX}_{\mathrm{RR}} \mathrm{X}_{\mathrm{EDR}} \mathrm{AR}$ models respectively.

Table 1: Performance comparison using the goodness of fit values between the trivariate (ARXXAR) models developed with respiration and with EDR.

\begin{tabular}{|c|c|c|c|c|c|c|c|}
\hline \multirow{2}{*}{$\begin{array}{l}\text { QT } \\
\text { dynamic } \\
\text { s }\end{array}$} & \multirow{2}{*}{$\begin{array}{l}\text { Model } \\
\text { Type }\end{array}$} & \multicolumn{3}{|c|}{ Fantasia Database } & \multicolumn{3}{|c|}{ Drivedb database } \\
\hline & & Young & Old & p value & No stress & Stressed & $p$ value \\
\hline \multirow[t]{2}{*}{$Q T_{\text {end }}$} & $A R X_{R R} X_{R E S P} A R$ & $\begin{array}{l}0.56(0.52- \\
0.59)\end{array}$ & $\begin{array}{l}0.54(0.49- \\
0.59)\end{array}$ & 0.41 & $\begin{array}{l}0.61(0.57- \\
0.64)\end{array}$ & $\begin{array}{l}0.53^{\#}(0.48- \\
0.58)\end{array}$ & 0.01 \\
\hline & $A R X_{R R} X_{E D R} A R$ & $\begin{array}{l}0.56(0.52- \\
0.58)\end{array}$ & $\begin{array}{l}0.52(0.48- \\
0.56)\end{array}$ & 0.07 & $\begin{array}{l}0.60(0.57- \\
0.66)\end{array}$ & $\begin{array}{l}0.54^{\#}(0.50- \\
0.60)\end{array}$ & 0.04 \\
\hline \multirow[t]{2}{*}{$Q T_{\text {peak }}$} & $A R X_{R R} X_{R E S P} A R$ & $\begin{array}{l}0.58(0.56- \\
0.62)\end{array}$ & $\begin{array}{l}0.54 *(0.51- \\
0.59)\end{array}$ & 0.01 & $\begin{array}{l}0.62(0.57- \\
0.68)\end{array}$ & $\begin{array}{l}0.58(0.51- \\
0.67)\end{array}$ & 0.19 \\
\hline & $A R X_{R R} X_{E D R} A R$ & $\begin{array}{l}0.60(0.56- \\
0.65)\end{array}$ & $\begin{array}{l}0.53 *(0.50- \\
0.57)\end{array}$ & 0.003 & $\begin{array}{l}0.63(0.55- \\
0.69)\end{array}$ & $\begin{array}{l}0.60(0.51- \\
0.65)\end{array}$ & 0.12 \\
\hline
\end{tabular}

All model fit values are given as median (first quartile-third quartile).

$\mathrm{QT}_{\text {end }}$ : Interval between $Q$ wave onset to $T$ wave end.

$\mathrm{QT}_{\text {peak }}$ : Interval between $Q$ wave onset to $T$ wave peak.

* indicates significant difference from Young group.

\# indicates significant difference in model fit from No stressed condition. 


\section{Discussion}

In this study, we have analysed the performance of EDR as an exogenous input for modeling VR dynamics using a linear parametric model from short-term ECG signal. Short-term HRV at resting condition and respiratory movement were reported to be linear with variation of $Q T$ interval [21,25]. Therefore, we used linear parametric autoregressive models to analyse the effect of respiration and HR on VR dynamics. The exogenous effect of respiration on $Q T$ variability was first reported by Porta et al. [26,2] and the authors have concluded that respiratory related artifacts directly modulate the $Q T$ interval. The cyclical modulation of VR process by respiration and the presence of synchronized high frequency content of $R T$ or $Q T$ interval with respiration even in the absence of $R R$ variability were also reported in various studies $[9,18]$. These findings proved the relationship between respiration and VR and justified the use of respiration or EDR as an exogenous input with $R R$ in the linear model structure of our study to predict VR dynamics. Previous studies reported use of both $\mathrm{QT}_{\text {peak }}$ and $\mathrm{QT}_{\text {end }}$ intervals with $R R$ intervals for model based analysis of VRV and VR dynamics [2,26]. Although Almeida et al. [2] emphasized the importance of considering $\mathrm{QT}_{\text {end }}$ in $Q T-R R$ modeling for complete description of VR dynamics, proper detection of $T$ wave end is disputable [26]. Therefore, both $\mathrm{QT}_{\text {peak }}$ and $\mathrm{QT}_{\text {end }}$ intervals were used in this study to model VR dynamics.

Model fit values significantly $(\mathrm{p}<0.05)$ increased with the inclusion of respiration or EDR for both case studies (i.e. Fantasia and drivedb databases) and for both $\mathrm{QT}_{\text {peak }}$ and $\mathrm{QT}_{\text {end }}$ dynamics models (Figure 4 and 5). This supports the previous findings that respiration affects $Q T$ dynamics $[9,26]$ and therefore, use of respiration or EDR in modeling establishes the importance of respiration for better comprehension and prognosis of VR dynamics in healthy subjects. Although model complexity (i.e. model order) was found insignificantly different $(\mathrm{p}>0.05)$ among the three types of models, $Q T-R R$ model needs relatively higher model order (average model order 17) than that of the models incorporating respiratory information (average model order 15). According to the theory of system identification, if addition of an exogenous input significantly increases the model fit without any significant increase in model complexity, then that input has 
important contribution in controlling the system dynamics [17]. Therefore, the reported significant increase in model fit values with the addition of respiratory information reflects the effect of respiration or EDR on VR dynamics rather than the effect of complex model structure.

EDR represents the modulatory effect of $R$ wave amplitude due to the rotation of mean cardiac electrical axis and the variation of thoracic impedance during respiratory cycle. It can reproduce respiratory information even with very low respiratory sinus arrhythmia (RSA) from surface ECG [20]. Although there is clear evidence of decreasing RSA with age [13], EDR still can represent the respiratory information necessary for describing respiratory effect on repolarization [20]. Similar result was found in our analysis, where the model fit values showed statistically insignificant difference between the use of respiratory signal and EDR for both case studies. These results validate the hypothesis that EDR can be used as a surrogate of respiratory information in modeling HRV and VRV interactions irrespective of age and psychological condition in healthy subjects.

Healthy ageing was found to be associated with the continual damage of integrated physiological regulatory system control. Increase in $Q T$ interval variability and reduction in vagal modulation with ageing was reported in healthy elderly population [23], which could cause the elderly subjects susceptible to diseases and make them unable to react properly to the perturbation of autonomic nervous system [12]. Moreover, RSA was reported to be decreased with ageing, which might affect the value of EDR [13] and consequently the model performance. These might be the reasons of the reported decrease in both types of model fit values (i.e. model with and without respiration information) in elderly population group compared to the young group (Table 1 and Figure 4 \& 5). Although the model fit values decrease with ageing, the model with respiration still performs better in describing VR dynamics than $Q T-R R$ model without respiration (Figure 4 and 5). Since no statistically significant difference in model fit values was found between respiration and EDR based models, it can be concluded that EDR can be used as a surrogate of respiration for modeling of VR dynamics in both young and elderly population. Another finding of this group's 
study is that the models incorporating respiration or EDR, which describe $\mathrm{QT}_{\text {peak }}$ dynamics can differentiate the Young and Elderly groups of Fantasia database (Table 1) while the $\mathrm{QT}_{\text {end }}$ dynamics models could not. This might be due to the absence of significant variability in $T_{p}-T_{e}$ interval (i.e. $T$ wave apex to $T$ wave end interval) dynamics in the subjects as $T_{p}-T_{e}$ interval variability was reported to increase with the alteration of psychological condition [29]. Since the ECG and respiration signals of Fantasia database were recorded in relaxed supine resting condition with no change in mental condition, the effect of $T_{p}-T_{e}$ interval variability was absent in the derived models.

Stress can induce temporal inhomogeneity in ventricular repolarization process that increases the complexity of $Q T-R R$ interaction [28]. Real life stressed situations like the driving of automobile in heavy traffic condition could alter the repolarization process by inducing $S T-T$ wave alteration [27]. Excessive stress could initiate arrhythmias through temporal and spatial dispersion of VR [16]. Therefore, decrease in model fitting values in our healthy young "Stressed" group (Table 1) could be due to the increase in sympathetic drive that might be the cause for the temporal dispersion in VR process. Models designed with EDR showed similar performance as of respiration signal in interpreting these changes in VR dynamics, which indicates that EDR can be used as a surrogate of respiration for modeling VR dynamics in psychologically stressed situations. Moreover, both respiration and EDR based models describing $\mathrm{QT}_{\text {end }}$ dynamics could significantly $(p<0.05)$ differentiate the "Stressed" and "No stress" groups, which might be linked with the effect of $T_{p}-T_{e}$ interval variability on VR dynamics.

\subsection{Limitations}

In this study, the respiration and ECG signals were recorded at supine resting condition for subjects in Fantasia database and in sitting condition within a car with seat belt fastened for the subjects of drivedb database. As a result, the effects of postural changes on EDR are not considered in this study that might affect the EDR based model performance. Therefore, we recommend the use of respiration signal in clinical settings and EDR should be used where respiration signal is absent. 
Although the fit value increases significantly with the addition of EDR or respiration in the $Q T-R R$ model, the fit values are not very high (Table 1). In this study, we assumed linear relation among respiration, HRV and VRV. However, these are not completely linear in nature and therefore, a more complex and non-linear model may improve the fitting value of the models describing VR dynamics. In addition, we assumed that respiration and $R R$ interval are completely independent of each other on their effect on VRV. This could be another reason for low fit value obtained in this study.

\section{Conclusion}

Irrespective of age and psychological conditions of the healthy individual, the derived linear parametric $Q T-R R-E D R$ models from short term ECG showed almost the same level of predictability as that of the models derived using respiration (i.e. $Q T-R R-R E S P$ ). Therefore, the proposed hypothesis that EDR can be used as a surrogate of respiration signal in $Q T-R R$ modeling from short term ECG segment recorded in steady postural condition (i.e. supine or sitting) was found to be correct in this study. Since EDR can be collected from the ECG signal only, this would reduce the complexity involved in recording respiration signal. Whether respiration or EDR is used, the model prediction capability showed significant improvement in comparison to normal $Q T-R R$ model without any respiratory information. Respiratory information based models can also significantly differentiate the effect of ageing and psychological changes due to stress of healthy subjects. In future, it will be interesting to explore the performance of EDR for modeling VR dynamics in controlled breathing conditions, various postural maneuvers and diverse pathological conditions.

References

1. Akaike H (1974) A new look at the statistical model identification. Automatic Control, IEEE Transactions on 19 (6):716-723

2. Almeida R, Gouveia S, Rocha AP, Pueyo E, Martinez JP, Laguna P (2006) QT variability and HRV interactions in ECG: quantification and reliability. IEEE transactions on bio-medical engineering 53 (7):1317-1329. doi:10.1109/TBME.2006.873682

3. Bailon R, Sornmo L, Laguna P (2006) A robust method for ECG-based estimation of the respiratory frequency during stress testing. IEEE transactions on bio-medical engineering 53 (7):1273-1285. doi:10.1109/TBME.2006.871888 
4. Berntson GG, Cacioppo JT, Quigley KS (1993) Respiratory sinus arrhythmia: autonomic origins, physiological mechanisms, and psychophysiological implications. Psychophysiology 30 (2):183-196

5. Boyle J, Bidargaddi N, Sarela A, Karunanithi M (2009) Automatic detection of respiration rate from ambulatory single-lead ECG. IEEE transactions on information technology in biomedicine : a publication of the IEEE Engineering in Medicine and Biology Society 13 (6):890-896.

doi:10.1109/TITB.2009.2031239

6. Clifford G, McSharry P, Tarassenko L (2002) Characterizing artefact in the normal human 24hour RR time series to aid identification and artificial replication of circadian variations in human beat to beat heart rate using a simple threshold. Computers in Cardiology, IEEE Computer Society press:129-132

7. de Chazal P, Heneghan C, Sheridan E, Reilly R, Nolan P, O'Malley M (2003) Automated processing of the single-lead electrocardiogram for the detection of obstructive sleep apnoea. IEEE transactions on bio-medical engineering 50 (6):686-696. doi:10.1109/TBME.2003.812203

8. Goldberger AL, Amaral LA, Glass L, Hausdorff JM, Ivanov PC, Mark RG, Mietus JE, Moody GB, Peng C-K, Stanley HE (2000) Physiobank, physiotoolkit, and physionet components of a new research resource for complex physiologic signals. Circulation 101 (23):e215-e220

9. Hanson B, Gill J, Western D, Gilbey MP, Bostock J, Boyett MR, Zhang H, Coronel R, Taggart $\mathrm{P}$ (2012) Cyclical modulation of human ventricular repolarization by respiration. Frontiers in physiology 3:379. doi:10.3389/fphys.2012.00379

10. Healey JA, Picard RW (2005) Detecting stress during real-world driving tasks using physiological sensors. Intelligent Transportation Systems, IEEE Transactions on 6 (2):156-166

11. Huikuri HV, Valkama JO, Airaksinen KEJ, Seppanen T, Kessler KM, Takkunen JT, Myerburg RJ (1993) Frequency-Domain Measures of Heart-Rate-Variability before the Onset of Nonsustained and Sustained Ventricular-Tachycardia in Patients with Coronary-Artery Disease. Circulation 87 (4):1220-1228

12. Iyengar N, Peng CK, Morin R, Goldberger AL, Lipsitz LA (1996) Age-related alterations in the fractal scaling of cardiac interbeat interval dynamics. Am J Physiol-Reg I 271 (4):R1078R1084

13. Kaushal P, Taylor JA (2002) Inter-relations among declines in arterial distensibility, baroreflex function and respiratory sinus arrhythmia. J Am Coll Cardiol 39 (9):1524-1530. doi:Doi 10.1016/S0735-1097(02)01787-4

14. Khaled ZB, Farges G (1992) First approach for respiratory monitoring by amplitude demodulation of the electrocardiogram. 14th Annual International Conference of the IEEE Engineering in Medicine and Biology Society, 6:2535-2536. doi:10.1109/IEMBS.1992.5761575

15. Khandoker AH, Karmakar CK, Palaniswami M (2009) Automated recognition of patients with obstructive sleep apnoea using wavelet-based features of electrocardiogram recordings. Comput Biol Med 39 (1):88-96. doi:DOI 10.1016/j.compbiomed.2008.11.003

16. Lampert R, Shusterman V, Burg MM, Lee FA, Earley C, Goldberg A, McPherson CA, Batsford WP, Soufer R (2005) Effects of psychologic stress on repolarization and relationship to autonomic and hemodynamic factors. J Cardiovasc Electr 16 (4):372-377. doi:DOI 10.1046/j.1540-8167.2005.40580.x

17. Lennart L (1999) System identification: theory for the user. Prentice Hall PTR, USA,

18. Lombardi F, Sandrone G, Porta A, Torzillo D, Terranova G, Baselli G, Cerutti S, Malliani A (1996) Spectral analysis of short term R-Tapex interval variability during sinus rhythm and fixed atrial rate. European heart journal 17 (5):769-778 
19. Malik M, Hnatkova K, Novotny T, Schmidt G (2008) Subject-specific profiles of QT/RR hysteresis. American journal of physiology Heart and circulatory physiology 295 (6):H2356-2363. doi:10.1152/ajpheart.00625.2008

20. Moody GB, Mark RG, Bump MA, Weinstein JS, Berman AD, Mietus JE, Goldberger AL (1986) Clinical validation of the ECG-derived respiration (EDR) technique. Computers in Cardiology, IEEE Computer Society press (13):507-510

21. Noriega M, Martinez JP, Laguna P, Bailon R, Almeida R (2012) Respiration effect on waveletbased ECG T-wave end delineation strategies. IEEE transactions on bio-medical engineering 59 (7):1818-1828. doi:10.1109/TBME.2011.2157824

22. Pan J, Tompkins WJ (1985) A real-time QRS detection algorithm. Biomedical Engineering, IEEE Transactions on (3):230-236

23. Piccirillo G, Cacciafesta M, Lionetti M, Nocco M, Di Giuseppe V, Moise A, Naso C, Marigliano V (2001) Influence of age, the autonomic nervous system and anxiety on QT-interval variability. Clin Sci 101 (4):429-438. doi:Doi 10.1042/Cs20000310

24. Porta A, Baselli G, Caiani E, Malliani A, Lombardi F, Cerutti S (1998) Quantifying electrocardiogram RT-RR variability interactions. Med Biol Eng Comput 36 (1):27-34. doi:Doi 10.1007/Bf02522854

25. Porta A, Guzzetti S, Furlan R, Gnecchi-Ruscone T, Montano N, Malliani A (2007) Complexity and nonlinearity in short-term heart period variability: comparison of methods based on local nonlinear prediction. IEEE transactions on bio-medical engineering 54 (1):94-106.

doi:10.1109/TBME.2006.883789

26. Porta A, Tobaldini E, Gnecchi-Ruscone T, Montano N (2010) RT variability unrelated to heart period and respiration progressively increases during graded head-up tilt. American journal of physiology Heart and circulatory physiology 298 (5):H1406-1414.

doi:10.1152/ajpheart.01206.2009

27. Simonson E, Baker C, Burns N, Keiper C, Schmitt OH, Stackhou.S (1968) Cardiovascular Stress (Electrocardiographic Changes) Produced by Driving an Automobile. Am Heart J 75 (1):125-\&. doi:Doi 10.1016/0002-8703(68)90123-3

28. Taggart P, Batchvarov VN, Sutton P, Young G, Young S, Patterson D (2009) Repolarization Changes Induced by Mental Stress in Normal Subjects and Patients with Coronary Artery Disease: Effect of Nitroglycerine. Psychosom Med 71 (1):23-29. doi:Doi 10.1097/Psy.0b013e31818a1d56

29. Taggart P, Sutton P, Redfern C, Batchvarov VN, Hnatkova K, Malik M, James U, Joseph A (2005) The effect of mental stress on the non-dipolar components of the T wave: Modulation by hypnosis. Psychosom Med 67 (3):376-383. doi:DOI 10.1097/01.psy.0000160463.10583.88

30. Yamada A, Hayano J, Horie K, Ieda K, Mukai S, Yamada M, Fujinami T (1993) Regulation of QT interval during postural transitory changes in heart rate in normal subjects. The American journal of cardiology 71 (11):996-998

31. Zarrini M, Sadr A (2009) Detecting T-wave using separated beats by adaptive threshold. Second International Conference on Computer and Electrical Engineering, ICCEE'09 1:323-326 


\section{University Library}

\section{- M I N E R VA}

\section{A gateway to Melbourne's research publications}

Minerva Access is the Institutional Repository of The University of Melbourne

\section{Author/s:}

Imam, MH;Karmakar, CK;Khandoker, AH;Palaniswami, M

Title:

Effect of ECG-derived respiration (EDR) on modeling ventricular repolarization dynamics in different physiological and psychological conditions

\section{Date:}

2014-10-01

\section{Citation:}

Imam, M. H., Karmakar, C. K., Khandoker, A. H. \& Palaniswami, M. (2014). Effect of ECGderived respiration (EDR) on modeling ventricular repolarization dynamics in different physiological and psychological conditions. MEDICAL \& BIOLOGICAL ENGINEERING \& COMPUTING, 52 (10), pp.851-860. https://doi.org/10.1007/s11517-014-1188-0.

Persistent Link:

http://hdl.handle.net/11343/283118 